Channeling maps for Si ions in Si : Assessing the binary collision approximation

Hobler, G.

2019-06-15

Hobler , G \& Nordlund, K 2019 , ' Channeling maps for Si ions in Si : Assessing the binary collision approximation ' , Nuclear Instruments \& Methods in Physics Research. Section B:

Beam Interactions with Materials and Atoms, vol. 449 , pp. 17-21 . https://doi.org/10.1016/j.nimb.2019.04.029

http://hdl.handle.net/10138/308759

https://doi.org/10.1016/j.nimb.2019.04.029

cc_by_nc_nd

acceptedVersion

Downloaded from Helda, University of Helsinki institutional repository.

This is an electronic reprint of the original article.

This reprint may differ from the original in pagination and typographic detail.

Please cite the original version. 
Authors preprint of paper published as Nuclear Instruments \& Methods B: 449 (2019), p. 17-21

\title{
Channeling maps for Si ions in Si: Assessing the binary collision approximation
}

\author{
G. Hobler ${ }^{\mathrm{a}, *}$ K. Nordlund ${ }^{\mathrm{b}}$ \\ ${ }^{a}$ Institute of Solid State Electronics, TU Wien, Gußhausstraße 25-25a, A-1040 Wien, Austria \\ ${ }^{b}$ Department of Physics, P. O. Box 43, FIN-00014 University of Helsinki, Finland
}

\begin{abstract}
Simulations based on the binary collision approximation (BCA) are in principle less accurate than molecular dynamics (MD) simulations. In this work, we present a comprehensive comparison between BCA and MD for Si ions impinging on a (001)-Si surface by comparing "channeling maps", i.e., projected ranges of the ions as a function of incidence direction in a representative part of the angular space. We find quantitative differences to develop as the energy decreases below $\sim 100 \mathrm{eV}$, but find qualitative agreement down to $\sim 10 \mathrm{eV}$. Moreover, the quality of the BCA channeling maps depends on the implementation of the $\mathrm{BCA}$, which is explained in terms of double-hits and missed collisions.
\end{abstract}

Keywords: channeling, binary collision approximation, molecular dynamics

\section{Introduction}

The slowing down of energetic ions in a crystal may be significantly reduced when the ions move along low-index crystallographic axes or planes. This so-called channeling

5 effect has long been used in ion beam analysis techniques such as RBS/channeling [1, and has received much attention in the semiconductor industry as it may significantly influence doping profiles introduced by ion implantation 2. More recently, channeling has been found to be relevant to ion irradiation of nanostructures, where it may ${ }_{40}$ lead to large variations of the sputtering yield [3].

A convenient means of characterizing channeling are "channeling maps" showing the projected range or nuclear energy deposition for a certain range of incidence directions. We have previously used molecular dynamics (MD) simulations and analytical approximations to calculate channeling maps for a variety of ion-target combinations [4, 5].

In the present work, we address the question whether accurate channeling maps may also be calculated using simulations based on the binary collision approximation ${ }_{50}$ (BCA), which run faster than MD and provide more detail than analytical theory. BCA simulations have been compared to MD results before [6, 7, 8, but only for selected conditions. The present paper goes beyond this older work the initial ion direction, are recorded. For the BCA simulations, the initial directions are chosen such that their stereographic projections lie on a Cartesian grid between the [001], [101], and [011] directions with 45 intervals in each direction. For the MD simulations the polar (azimuthal) angles are chosen as before [4, 5 in $1^{\circ}$ intervals in the range of $0^{\circ}$ to $89^{\circ}\left(90^{\circ}\right)$. Thus, a BCA channeling map requires $46 \times 46=2116$ simulations, while for an MD channeling map $90 \times 91=8190$ simulations are run. In each simulation 10000 ion trajectories for BCA and 5000 for MD are followed to obtain sufficient statistics. Only directions between [001], [101], [111], and [011] are taken into account when comparing BCA and MD. Other directions are equivalent to directions in this range except for surface effects, which are not the focus of the present investigation. For the calculation of differences between BCA and MD maps, the MD data are interpolated to the BCA directions by bilinear interpolation.

BCA simulations are performed with the current version of the IMSIL [9] code, while MDRANGE [10] is used for MD simulations. MDRANGE uses the recoil interaction approximation, i.e., only forces between the ion and target atoms are taken into account but no interactions among the target atoms. This approximation is thought as long as the ion energy is above a few eV. MDRANGE calculations are more accurate than BCA simulations as they take simultaneous interactions between the ion and target atoms into account.

Care has been taken to implement the same physical models consistently in both codes. The universal ZBL potential [11] is used with a cutoff radius of $3 \AA$ for MD and a cutoff impact parameter of $4.26 \AA$ for $\mathrm{BCA}$, both tested to be large enough to include all relevant interaction. The

* Corresponding author

Email address: gerhard.hobler@tuwien.ac.at (G. Hobler) 
electronic stopping power is described by

$$
S_{\mathrm{e}}=k k_{\mathrm{L}} E^{p}
$$

with $p=0.375, k_{\mathrm{L}}$ the Lindhard prefactor [12], and $k=$ $4.0175 \mathrm{eV}^{0.125}$, corresponding to the 1996 parameterization of the ZBL electronic stopping power [11]. In both codes electronic stopping is treated in a nonlocal manner and is set to zero when the ion is further away from the top atomic layer than $a_{\text {lat }} / 8$ in the direction towards the vacuum, where $a_{\text {lat }}$ denotes the lattice constant of $\mathrm{Si}$. In MD, electronic stopping is calculated from the ion veloc75 ity, i.e., the kinetic energy, while in BCA it is calculated from the kinetic energy before or after a collision. As the ion velocity is reduced during collisions, this should lead to lower electronic stopping in MD. MD test runs comparing the standard treatment with one using the sum of kinetic and potential energy showed that the difference is marginal, so the standard treatment was kept. The thermal displacements of atoms were calculated both in BCA and MD with the Debye model implemented with the Stegun handbook approximation as described in detail in Ref. ${ }^{\mathbf{1 2 0}}$ [5, with a Debye temperature of $493.6 \mathrm{~K}$.

A trajectory cutoff energy of $1 \mathrm{eV}$ is used in both types of simulation. Again, there is a difference between the standard treatments of trajectory cutoff in MD and standard BCA implementations: While in MD the kinetic en- ${ }^{\mathbf{1 2 5}}$ ergy is compared to the cutoff after each time step, in standard BCA only the energy before and after collisions are available for this comparison. Since the kinetic energy is reduced during collisions, this leads to earlier cutoffs in MD compared to BCA. However, it is possible to cal-130 culate the kinetic energy at the apsis of a collision, and we use this value in the cutoff energy criterion for BCA simulations. Moreover, provision was made in IMSIL to correctly calculate the length of the last free flight path of a trajectory if the ion energy falls below the cutoff energy ${ }^{\mathbf{1 3 5}}$

100 due to electronic stopping: Normally, free flight paths are chosen, and the ion is moved by that path length (or, alternatively, not moved at all) if the energy drops below the cutoff along the path. Instead, we calculate the last free flight path length by integration of Eq. (1). Both features ${ }^{140}$ 105 showed some effects at the lowest impact energies and have therefore been used in obtaining the results presented in Sect. 4 .

\section{BCA implementations}

In the $\mathrm{BCA}$, the ion-target interaction is described by isolated collisions of the ion with the target atoms. The trajectories are constructed from the asymptotes before and after the collisions. Special care has to be taken torso avoid double-hits of the same target atoms: In a straightforward implementation the ion is put into the intersection point of its incoming and outgoing asymptote (see Fig. 1 1a) 13. Since the crystal is reconstructed from its lattice sites after each collision, it is possible that the lattice site of the $\mathrm{IF5}_{155}$ a)

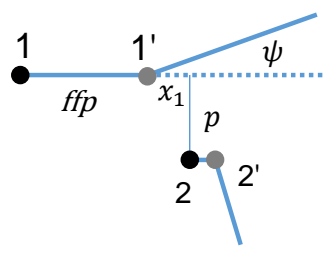

- 1,2 ... before collision

- 1',2' ... after collision

b)

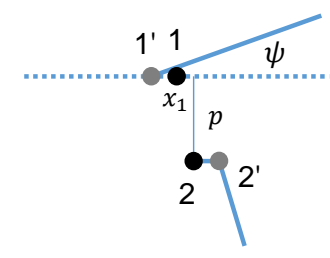

Figure 1: Asymptotes of the ion (1) and recoil (2) trajectories in a binary collision. The ion is assumed to initially move to the right. In the standard model, after the collision, ion and recoil are placed into the intersection points of incoming and outgoing asymptotes. This normally leads to positive free flight paths ffp (panel a), but can also lead to movement in the direction opposite to the initial ion momentum (panel b).

target atom just hit (2) is in front of the ion (1') measured along the new ion direction. To avoid selecting atom 2 again as a collision partner, one may demand a minimum free flight path for the next collision [13, move the ion forward along the new direction such that atom 2 is just not selected [14, 8, and/or exclude previous collision partners from the search [8]. In the current version of IMSIL we use the latter approach, where we remember a maximum of six old collision partners and clear the list if the scattering angle of the ion exceeds $30^{\circ}$. We note that the problem of double-hits does not occur as easily if the intersection point of the ion's asymptotes is assumed at the foot of the impact parameter on the incoming asymptote $\left(x_{1}=0\right.$ in Fig. 1), an approximation often used in BCA codes for amorphous targets such as TRIM [15]. This, however, does not provide the correct outgoing asymptote.

Another possible problem is illustrated in Fig. 1 b. At low energies the distance $x_{1}$ between the intersection point and the foot of the impact parameter may be quite large [16] so the ion may move in the direction opposite to its momentum, corresponding to negative free flight paths. This may lead to artifacts in the calculation of atom displacement in amorphous materials [17]. In Ref. [17] it was proposed not to move the ion if the length of the free flight path is calculated to be negative.

In this work, four implementations of the BCA are investigated:

Model 1: The ion is placed at the intersection point of its asymptotes, and up to six old collision partners are excluded from the next collision. The list is cleared when the ion's scattering angle exceeds $30^{\circ}$ ("IMSIL default").

Model 2: As Model 1. but only the last collision partner is remembered ("multihit").

Model 3: As Model 1, but the ion is placed at the foot of the impact parameter on the incoming asymptote $\left(x_{1}=0\right)$ after the collision ("no $\tau$ ").

Model 4: As Model 1, but only non-negative free flight 
Table 1: Theoretical minimum energies for channeling $\left(E_{\min }\right)$ of $\mathrm{Si}$ ions in Si for all axial channels with $E_{\min }<1 \mathrm{keV}$ and all planar channels with $E_{\min }<100 \mathrm{keV}$.

\begin{tabular}{cc} 
channel & $E_{\min }(\mathrm{eV})$ \\
\hline$\langle 100\rangle$ & 268 \\
$\langle 110\rangle$ & 24 \\
$\langle 111\rangle$ & 683 \\
$\langle 211\rangle$ & 194 \\
$\langle 321\rangle$ & 545 \\
$\langle 431\rangle$ & 948
\end{tabular}

\begin{tabular}{cc} 
channel & $E_{\min }(\mathrm{eV})$ \\
\hline$\{100\}$ & 24607 \\
$\{110\}$ & 3592 \\
$\{111\}$ & 106 \\
$\{311\}$ & 57067
\end{tabular}

paths are allowed ("ffp $>0$ ").

\section{Results and Discussion}

Channeling maps for $10 \mathrm{keV}, 1 \mathrm{keV}, 100 \mathrm{eV}, 50 \mathrm{eV}$, $30 \mathrm{eV}$, and $10 \mathrm{eV}$ Si ions in Si are shown in Fig. 2. Chan-215 ing indicated by increased values of the mean range compared to its lowest values (dark blue color). In the upper left part (triangle [001]-[111]-[011]) the MD results are depicted, while the lower right part (triangle [001][101]-[111]) shows the BCA results using Model 1 (IMSIL220 Due to the crystal symmetry the BCA results should be mirror reflections of the MD results at the (1 10$)$ plane in case of perfect agreement between BCA and MD. Good qualitative agreement regarding the existence and width of the various channels is found for all energies with?25 the quantitative error increasing, as expected, with decreasing energy. Noticable differences are found at $100 \mathrm{eV}$ and lower energies.

There is also excellent agreement with minimum energies for channeling $E_{\min }$ as calculated by channeling theory ${ }^{\mathbf{2 3 0}}$ 4, 18. The values are shown in Tab. 1. In the channeling map for the highest energy (10 keV), many axial channels but only the (111) and $\{110\}$ planar channels are visible. The number of axial channels is reduced at $1 \mathrm{keV}$ to those given in Tab.1. At $100 \mathrm{eV}$ and down to $30 \mathrm{eV}$ only the [101/235 and [011] channels are active, in agreement with Tab. 1.

In both the MD and BCA results, the $\langle 110\rangle$ channels appear to move away from their crystallographic directions as the energy is decreased. This can be explained by a surface effect as illustrated in Fig. 3. If an ion approacheseso the (001) surface at an angle $\theta$ close to the $\langle 110\rangle$ channeling direction, if it heads towards the channel center, and if its energy is low enough that it may effectively interact with atoms on all sides of the channel when inside the channel, its first collision with a target atom will scatter it away from the surface normal, thus increasing $\theta$. The perfect channeling direction inside the target $\left(\theta=45^{\circ}\right)$ therefore $_{245}$ is obtained with an incidence angle of $\theta<45^{\circ}$. Since in the channeling maps the initial ion direction is used, in Fig. 2 the most likely channeling direction appears to be shifted towards the surface normal for $100 \mathrm{eV}$ and $50 \mathrm{eV}$. The effect goes away at higher energies since single collisions ${ }_{250}$ are less important there.
Another artifact of the surface is the apparent reappearance of the [111] channel at energies less than $100 \mathrm{eV}$. At low energies, the fraction of reflected ions increases for larger incidence angles $\theta$. While the reflected ions are disregarded in the mean range calculation, also more ions are scattered into directions parallel to the surface without penetrating into the target. They experience less stopping than if they travelled in the bulk and thus have a larger range.

The BCA simulations have been repeated with all four models described in Sect. 3. For each model, energy, and direction within the channeling maps, the difference between BCA and MD range has been calculated. The range of difference within each map is depicted in Fig. 4 as a function of energy for each model. Generally, at low energies the magnitude of the difference increases with decreasing energy. For Models 1 and 2 ("IMSIL default" and "multihit") the BCA ranges are mostly smaller than the MD ranges with significantly better results for the "IMSIL default" model, which avoids double-hits of six previous collision partners. In Model 2 more double-hits occur with correspondingly larger energy transfer per path length, which results in smaller ranges.

When the ion is placed at the foot of the impact parameter on the incoming asymptote after the collision (Model 3), the ion is generally placed more in forward direction than with Models 1 and 2. Therefore there is a higher risc of missing collision partners, which leads to larger ranges. The difference with respect to the MD results is therefore positive at small energies. The same is true of Model 4 ("ffp $>0$ "): Here, backward motion of the ion is prevented, which reduces the chance of finding collision partners and therefore leads to an increase in the mean range.

Inspection of the maps of the range difference (Fig. 5 for $100 \mathrm{eV}$ ) indicates that at low energies particularly large magnitudes of the difference are found around the [112] direction. In (112) planes the lattice sites are arranged relatively closely packed on a rectangular grid, see Fig. 6. In large regions there are four atoms within the cutoff impact parameter, with which an ion moving in the [112] direction should interact simultaneously. This is the most critical situation for double-hitting or missing of collision partners in a BCA simulation. In contrast, in a [011] channel, there are typically only three atoms in (011) planes within the cutoff impact parameter whose interaction should be treated simultaneously.

\section{Conclusions}

We have shown excellent quantitative agreement between BCA and MD simulations for the transport of $\mathrm{keV}$ $\mathrm{Si}$ atoms in all directions of a Si crystal. At smaller energies (100 eV and below) some quantitative differences develop, while the qualitative features remain the same between BCA and MD. We have also shown that the quantitative agreement between $\mathrm{BCA}$ and $\mathrm{MD}$ depends on the 

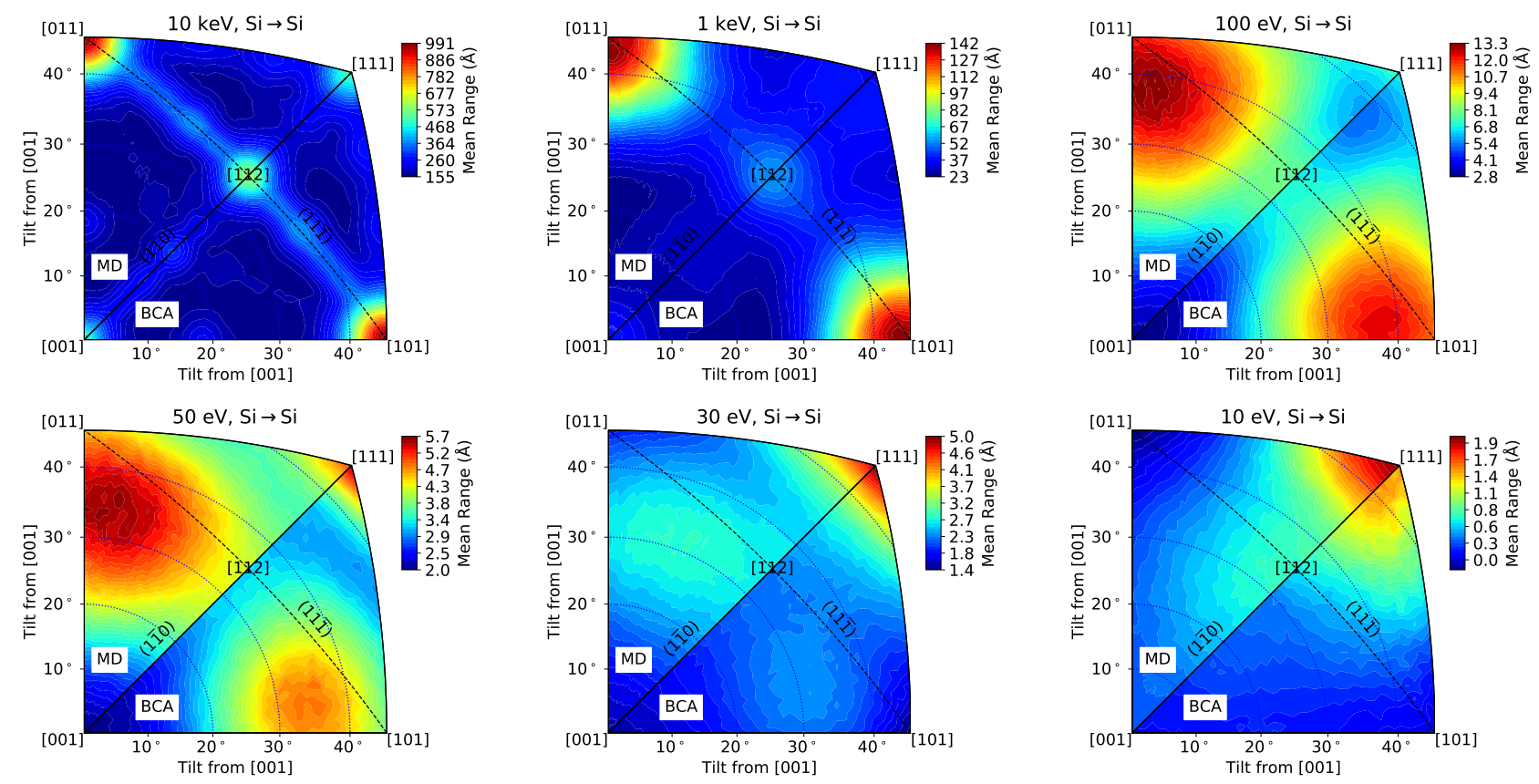

Figure 2: Comparison of channeling maps computed by MD (upper left half of each map) and BCA (lower right half) for $10 \mathrm{keV}, 1 \mathrm{keV}$, $100 \mathrm{eV}, 50 \mathrm{eV}, 30 \mathrm{eV}$, and $10 \mathrm{eV} \mathrm{Si}$ ions in $\mathrm{Si}$. Due to crystal symmetry the maps should be symmetric with respect to the (11̄0) plane. For the BCA simulations Model 1 (IMSIL default) has been used. The maps are shown as stereographic projections of the initial ion directions.

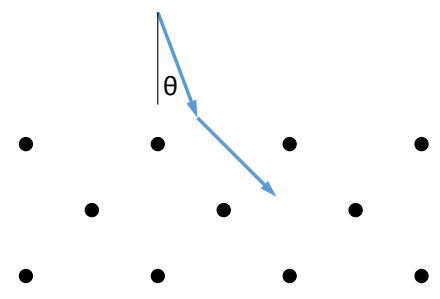

Figure 3: Illustration of the effect of the surface on the most likely channeling direction using a simplified lattice.

implementation of the BCA, and we have explained the differences in terms of double-hits and missed collisions.

It should be noted that more than the four BCA models investigated in this work exist. For instance, in a previous version of IMSIL 8 , the ion was moved forward along the outgoing asymptote such that a double-hit was avoided. Moreover, in the MARLOWE code [13] an algorithm for treating collisions simultaneously is implemented. Since these models require more substantial changes to our current code, we leave them for future consideration, as well as an investigation of the effects of ion and target atom mass and crystal structure. While the present study is not meant to be comprehensive, it does point out the impor-

\section{Acknowledgment}

KN acknowledges grants of computer capacity from the IT Centre for Science in Finland, CSC and the

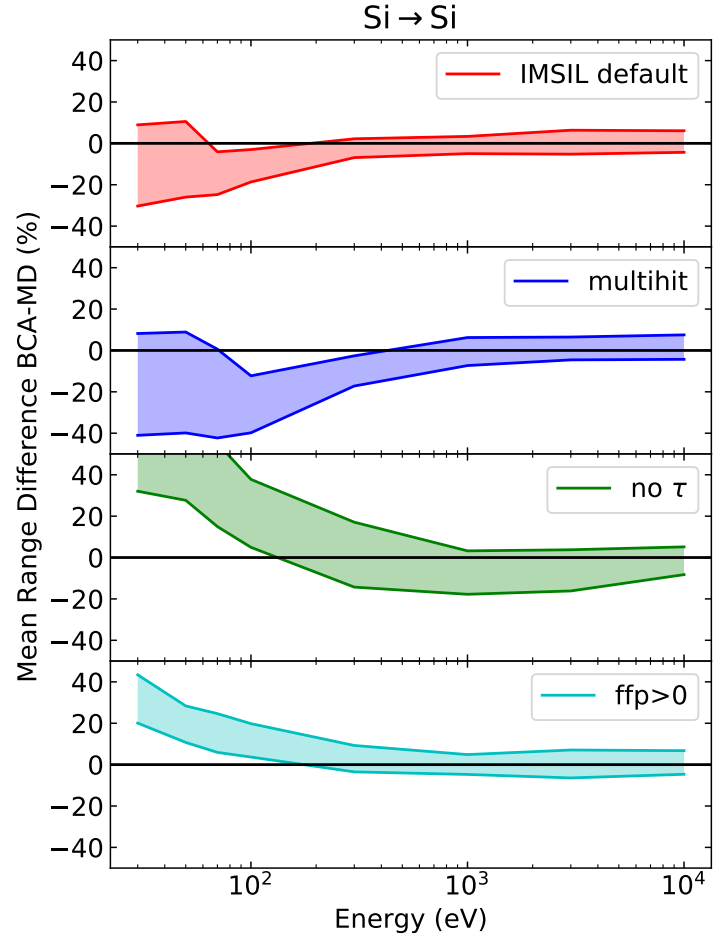

Figure 4: Difference between mean ranges calculated with BCA and MD for the four BCA models as a function of energy. The solid lines correspond to the minimum and maximum difference across the channeling map, the shaded areas indicate the ranges of the difference. 
Finnish Grid and Cloud Infrastructure (persistent identifier urn:nbn:fi:research-infras-2016072533).

\section{References}
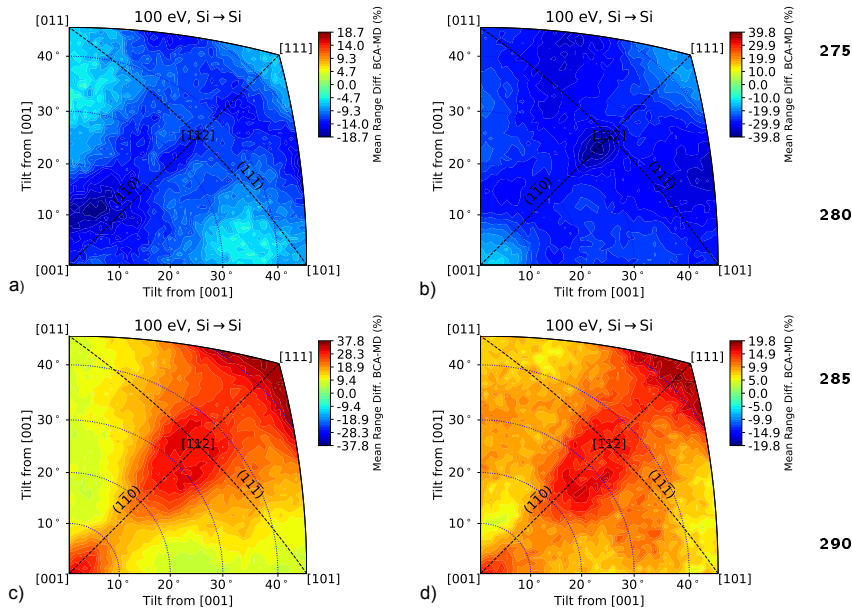

Figure 5: Channeling maps showing the difference between mean ranges obtained with $\mathrm{BCA}$ and $\mathrm{MD}$ simulations for $\mathrm{BCA}$ models ${ }_{295}$ 1-4: a) IMSIL default, b) multihit, c) no $\tau$, d) ffp $>0$.

[1] J. R. Tesmer, M. Nastasi, Handbook of Modern Ion Beam Materials Analysis, Materials Research Society, Pittsburgh, PA, 1995.

[2] M. Ameen, I. Berry, W. Class, H.-J. Gossmann, L. Rubin, Ion Implantation, in: R. Doering, Y. Nishi (Eds.), Handbook of Semiconductor Manufacturing Technology, 2nd Edition, CRC Press, Boca Raton, London, New York, 2008.

[3] J. A. Hinks, F. Hibberd, K. Hattar, A. Ilinov, D. C. Bufford, F. Djurabekova, G. Greaves, A. Kuronen, S. E. Donnelly, K. Nordlund, Effects of crystallographic and geometric orientation on ion beam sputtering of gold nanorods, Sci. Rep. 8 (1) (2018) 512. doi:10.1038/s41598-017-17424-9

[4] K. Nordlund, F. Djurabekova, G. Hobler, Large fraction of crystal directions leads to ion channeling, Phys. Rev. B 94 (21) (2016) 214109. doi:10.1103/PhysRevB.94.214109

[5] K. Nordlund, G. Hobler, Dependence of ion channeling on relative atomic number in compounds, Nucl. Instrum. Meth. Phys. Res. Bdoi:10.1016/j.nimb.2017.11.020

[6] P. Sigmund, M. T. Robinson, M. I. Baskes, M. Hautala, F. Z. Cui, W. Eckstein, Y. Yamamura, S. Hosaka, T. Ishitani, V. I. Shulga, D. E. Harrison, I. R. Chakarov, D. S. Karpuzov, E. Kawatoh, R. Shimizu, S. Valkealahti, R. M. Nieminen, G. Betz, W. Husinsky, M. H. Shapiro, M. Vicanek, H. M. Urbassek, Round robin computer simulation of ejection probability in sputtering, Nucl. Instrum. Meth. Phys. Res. B 36 (1989) 110-123.

[7] K. Gärtner, D. Stock, B. Weber, G. Betz, M. Hautala, G. Hobler, M. Hou, S. Sarite, W. Eckstein, J. J. JimenezRodriguez, A. M. C. Perez-Martin, E. P. Andribet, V. Konoplev, A. Gras-Marti, M. Posselt, M. H. Shapiro, T. A. Tombrello, H. M. Urbassek, H. Hensel, Y. Yamamura, W. Takeuchi, Round robin computer simulation of ion transmission through thin crystalline layers, Nucl. Instrum. Meth. Phys. Res. B 102 (1995) 183-197. doi:10.1016/0168-583X (95)0489-0

[8] G. Hobler, G. Betz, On the useful range of application of molecular dynamics simulations in the recoil interaction approximation, Nucl. Instrum. Meth. Phys. Res. B 180 (1âĂS4) (2001) 203-208. doi:10.1016/S0168-583X(01)00418-9.

[9] G. Hobler, Monte Carlo simulation of two-dimensional implanted dopant distributions at mask edges, Nucl. Instrum.

Meth. Phys. Res. B 96 (1995) 155-162. doi:10.1016/ 0168-583X (94)00476-5.

[10] K. Nordlund, Molecular dynamics simulation of ion ranges in

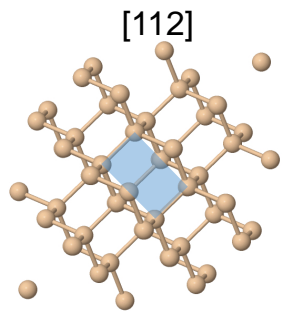

[011]

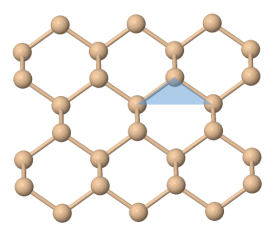

Figure 6: Si diamond lattice viewed in the [112] and [011] direction. The shaded rectangle and triangle connect nearest lattice sites in (112) and (011) planes, respectively.

315 the 1-100 keV energy range, Comp. Mater. Sci. 3 (1995) 448456.

[11] J. F. Ziegler, J. P. Biersack, U. Littmark, The Stopping and Range of Ions in Solids, Pergamon Press, New York, 1985.

[12] J. Lindhard, M. Scharff, Energy dissipation by ions in the $\mathrm{keV}$ region, Phys. Rev. 124 (1) (1961) 128-130.

[13] M. T. Robinson, I. M. Torrens, Computer simulation of atomicdisplacement cascades in solids in the binary-collision approximation, Phys. Rev. B 9 (12) (1974) 5008-5024.

[14] G. Hobler, G. Fehlmann, A study of ultra-shallow implanted dopant profiles in silicon using BC and MD simulations, Radiat. Eff. Def. Sol. 141 (1-4) (1997) 113-125. doi:10.1080/ 10420159708211562

[15] J. P. Biersack, L. G. Haggmark, A Monte Carlo computer program for the transport of energetic ions in amorphous targets, Nucl. Instr. Meth. 174 (1980) 257-269.

[16] W. Eckstein, Computer Simulation of Ion-Solid Interactions, Springer, Berlin, 1991.

[17] G. Hobler, D. Maciążek, Z. Postawa, Ion bombardment induced atom redistribution in amorphous targets: $M D$ versus $\mathrm{BCA}$, $10.1016 / j . n i m b .2019 .03 .028$ 
[18] G. Hobler, Critical angles and low-energy limits to ion channeling in silicon, Radiat. Eff. Def. Sol. 139 (1996) 21-85. 